\title{
Rotational properties of trapped bosons
}

\author{
F. Brosens and J. T. Devreese* \\ Departement Natuurkunde, Universiteit Antwerpen (UIA), Universiteitsplein 1, B-2610 Antwerpen, Belgium \\ L. F. Lemmens \\ Departement Natuurkunde, Universiteit Antwerpen (RUCA), Groenenborgerlaan 171, B-2020 Antwerpen, Belgium
}

(Received 3 September 1996; revised manuscript received 8 November 1996)

Based on the Hellman-Feynman theorem, it is shown that the average square radius of a cloud of interacting bosons in a parabolic well can be derived from their free energy. As an application, the temperature dependence of the moment of inertia of noninteracting bosons in a parabolic trap is determined as a function of the number of bosons. Well below the critical condensation temperature, the Bose-Einstein statistics are found to substantially reduce the moment of inertia of this system, as compared to a gas of "distinguishable" particles in a parabolic well. [S1050-2947(97)00703-8]

PACS number(s): 03.75.Fi, 05.30.Jp, 32.80.Pj

The study of a crowd of atoms in the same quantum state has gained great importance since the recent observation of the Bose-Einstein condensation of magnetically trapped gases of rubidium [1], sodium [2], and, albeit only with indirect evidence, lithium [3]. According to Stringari [4], who draws a parallel with superfluid systems [5], the rotational properties of a confined Bose gas provide a natural way to analyze the deviations from rigid motion due to condensation. He suggested that condensation effects of magnetically trapped gases should be observable in their moment of inertia, which he calculated approximately from the linear response properties of a Bose gas of harmonic oscillators without two-body interactions, relying on the semiclassical approximation [6-8] for the excited states.

Using the Hellman-Feynman theorem, we show in the present paper that the moment of inertia of a confined Bose gas can be derived directly from the dependence of its free energy on the strength of the confining parabolic potential, even if two-body interactions are included. Indeed, for the Hamiltonian

$$
\begin{aligned}
\mathcal{H} & =\sum_{j=1}^{N} \frac{p_{j}^{2}}{2 m}+\sum_{j<l}^{N} v\left(\vec{r}_{j}-\vec{r}_{l}\right)+\frac{m}{2} \sum_{j}^{N}\left[\omega_{z}^{2} z_{j}^{2}+\omega^{2}\left(x_{j}^{2}+y_{j}^{2}\right)\right] \\
& =\mathcal{H}_{\text {kin }}+\mathcal{H}_{\text {int }}+\mathcal{H}_{\text {conf }}
\end{aligned}
$$

it is straightforward to show that the confinement contribution $\mathcal{U}_{\text {conf }} \equiv\left\langle\mathcal{H}_{\text {conf }}\right\rangle$ to the internal energy can be written as

$$
\mathcal{U}_{\text {conf }}=\frac{1}{\mathcal{Z}} \operatorname{Tr}\left(e^{-\beta \mathcal{H}} \mathcal{H}_{\text {conf }}\right)=\frac{m}{2} N\left(\omega_{z}^{2}\left\langle z^{2}\right\rangle+\omega^{2}\left\langle x^{2}+y^{2}\right\rangle\right)
$$

where $\mathcal{Z}$ is the partition function. Denoting the free energy by $\mathcal{F}$, the Hellman-Feynman theorem states that

\footnotetext{
*Also at Universiteit Antwerpen (RUCA) and Eindhoven University of Technology, Eindhoven, The Netherlands.
}

$$
\mathcal{U}_{\mathrm{conf}}=\omega_{z}^{2} \frac{\partial \mathcal{F}}{\partial\left(\omega_{z}^{2}\right)}+\omega^{2} \frac{\partial \mathcal{F}}{\partial\left(\omega^{2}\right)}
$$

relating thereby the average square radius $\left\langle\rho^{2}\right\rangle \equiv\left\langle x^{2}+y^{2}\right\rangle$ of the system in the $x-y$ plane (and hence its moment of inertia with respect to the $z$ axis) to partial derivatives of the free energy. This relation is valid both for distinguishable and for identical particles. If the free energy exhibits critical behavior, it will also be reflected in the average square radius of the Bose gas and consequently in its rotational properties.

In order to estimate the moment of inertia quantitatively, we consider for simplicity an ideal cloud of noninteracting bosons trapped in a parabolic well characterized by a frequency $w$. With $\mu$ denoting the chemical potential and $\beta=1 / k T$, where $k$ is Boltzmann's constant and $T$ the temperature, the partition function in the grand-canonical ensemble becomes

$$
\begin{gathered}
\Xi_{\mu}=\prod_{\nu=0}^{\infty}\left(\frac{1}{1-e^{-\beta\left(\epsilon_{\nu}-\mu\right)}}\right)^{(1 / 2)(\nu+1)(\nu+2)}, \\
\epsilon_{\nu}=\left(\nu+\frac{3}{2}\right) \hbar w, \quad \nu=0,1,2, \ldots,
\end{gathered}
$$

where $\frac{1}{2}(\nu+1)(\nu+2)$ is the number of different possibilities to occupy the $\nu$ th level. This multiplicity depends on the symmetry and is considered here for the isotropic case $\omega_{z}^{2}=\omega^{2}$. For cylindrical symmetry, e.g., it would be 1 along the symmetry axis and $\nu+1$ in the plane orthogonal to the symmetry axis.

The expression (4) is the isotropic limit of the grand canonical partition function for a Bose gas of anisotropic oscillators [9], from which the subsequent derivation can be generalized to the anisotropic case $\omega_{z}^{2} \neq \omega^{2}$. The subsequent discussion is limited to the isotropic Bose gas. The groundstate occupancy and the thermodynamic properties including the anisotropic case have recently been analyzed in several papers [9-11] and are currently also the subject of several works [12-14]. 
The average number of bosons $N=(1 / \beta)(\partial / \partial \mu) \ln \Xi_{\mu}$ and their internal energy $U=-\partial \ln \Xi_{\mu} / \partial \beta+N \mu$ in an isotropic parabolic confining potential are

$$
\begin{gathered}
N=\sum_{\nu=0}^{\infty} n_{\nu}, \quad U=\sum_{\nu=0}^{\infty} n_{\nu} \epsilon_{\nu}, \\
n_{\nu}=\frac{1}{2}(\nu+1)(\nu+2) \frac{e^{-\beta\left(\epsilon_{\nu}-\mu\right)}}{1-e^{-\beta\left(\epsilon_{\nu}-\mu\right)}},
\end{gathered}
$$

where $n_{\nu}$ is the occupation number of the $\nu$ th level. Furthermore, the Hellman-Feynman theorem leads to the average square radius of the boson cloud

$$
\begin{aligned}
\frac{1}{2} m N\left\langle\vec{r}^{2}\right\rangle & =-\frac{1}{\beta} \frac{d}{d\left(w^{2}\right)} \ln \Xi_{\mu} \\
& =\frac{1}{4 w^{2}} \sum_{\nu=0}^{\infty}(\nu+1)(\nu+2) \epsilon_{\nu} \frac{e^{-\beta\left(\epsilon_{\nu}-\mu\right)}}{1-e^{-\beta\left(\epsilon_{\nu}-\mu\right)}} \\
& =\frac{1}{2} \frac{U}{w^{2}} .
\end{aligned}
$$

Consequently, the averaged potential energy becomes $\frac{1}{2}$ $m w^{2} N\left\langle\vec{r}^{2}\right\rangle=\frac{1}{2} U$, as could have been anticipated from the virial theorem. The moment of inertia relative to the $z$ axis is therefore given by

$$
\Theta=m N\left\langle x^{2}+y^{2}\right\rangle=\frac{2}{3} \frac{U}{w^{2}},
$$

which is the basic result of the present paper. The critical behavior of the internal energy (and consequently in the specific heat) of the model is directly reflected in the rotational properties of the boson gas.

The evaluation of the internal energy for a given number $N$ of particles requires the knowledge of the chemical potential. By expressing the chemical potential as a function of the number of particles in the ground state

$$
n_{0}=\frac{e^{-\beta[(3 / 2) \hbar w-\mu]}}{1-e^{-\beta[(3 / 2) \hbar w-\mu]}} \Rightarrow e^{-\beta[(3 / 2) \hbar w-\mu]}=\frac{n_{0}}{n_{0}+1},
$$

the total number of particles $N$ is obtained in terms of $n_{0}$,

$$
N=\sum_{\nu=0}^{\infty} \frac{1}{2}(\nu+1)(\nu+2)\left(\frac{n_{0} e^{-\beta \hbar w \nu}}{n_{0}+1-n_{0} e^{-\beta \hbar \omega \nu}}\right) .
$$

However, as it turns out, this series is not very appropriate for numerical treatment. A much more efficient and numerically well-convergent expression results if each term in the denominator of Eq. (10) is expanded in powers of $e^{-\beta \hbar w \nu}$, which allows one to perform the summation over $\nu$. The same procedure can be used for the internal energy. The results can be written in the following form, which is numerically stable:

$$
\begin{aligned}
N & =\sum_{l=1}^{\infty}\left(\frac{n_{0}}{n_{0}+1}\right)^{l} \frac{1}{\left(1-e^{-\beta \hbar w l}\right)^{3}} \\
& =n_{0}+\sum_{l=1}^{\infty}\left(\frac{n_{0}}{n_{0}+1}\right)^{l}\left(\frac{1}{\left(1-e^{-\beta \hbar w l}\right)^{3}}-1\right), \\
U & =\frac{3}{2} \hbar w \sum_{l=1}^{\infty}\left(\frac{n_{0}}{n_{0}+1}\right)^{l} \frac{1+e^{-\beta \hbar w l}}{\left(1-e^{-\beta \hbar w l}\right)^{4}} \\
& =\frac{3}{2} \hbar w\left(N+2 \sum_{l=1}^{\infty}\left(\frac{n_{0}}{n_{0}+1}\right)^{l} \frac{e^{-\beta \hbar w l}}{\left(1-e^{-\beta \hbar w l}\right)^{4}}\right) .
\end{aligned}
$$

In these expressions, $l$ is the length of a cycle in the cyclic decomposition of the partition function [15] obtained from the symmetrized density matrix for $N$ oscillators without two-body interactions.

The high-temperature limit $\beta \rightarrow 0$ of the internal energy and of the depletion of the condensate can now be analyzed directly. There is no longer a need to make the continuum approximation by introducing a parametrized form for the density of states [9]. In this limit, and without making the continuum approximation, $N-n_{0}$ becomes

$$
\begin{aligned}
\frac{k T}{\hbar w} \gg 1 \Rightarrow N-n_{0} \approx & \sum_{l=1}^{\infty}\left(\frac{n_{0}}{n_{0}+1}\right)^{l}\left[\frac{1}{\hbar^{3} w^{3} \beta^{3}} \frac{1}{l^{3}}+\frac{1}{\hbar^{2} w^{2} \beta^{2}} \frac{3}{2 l^{2}}\right. \\
& \left.+O\left(\frac{1}{\beta}\right)\right],
\end{aligned}
$$

and similarly for the internal energy $U$. For $n_{0} \gg 1$, the summations yield

$$
\begin{aligned}
& N-n_{0} \approx N\left(\frac{T}{T_{c}}\right)^{3}\left(1+\frac{3 \zeta(2)}{2 \zeta(3)} \frac{\hbar w}{k T}\right)+O(T), \\
& \frac{U}{\frac{3}{2} \hbar w N} \approx 1+2\left(\frac{T}{T_{c}}\right)^{3}\left[\frac{k T}{\hbar w} \frac{\zeta(4)}{\zeta(3)}+1+O\left(\frac{1}{T}\right)\right],
\end{aligned}
$$

with $T_{c}=(\hbar w / k)[N / \zeta(3)]^{1 / 3}$. This result for $N-n_{0}$ has been derived (also without the continuum approximation) in [11] and coincides with the result within an improved continuum approximation as derived in $[9,11]$. The temperature expansion for the internal energy $U$ is obtained in [9] using the continuum approximation.

For arbitrary temperature, the ground-state occupancy $n_{0}$ and the internal energy $U$ have to be evaluated numerically from Eq. (11). This can be done efficiently by first solving for the quantity $n_{0} /\left(n_{0}+1\right)$, which is bracketed between 0 and 1 . The resulting ground-state occupancy clearly exhibits the onset of a phase transition near the transition temperature [8-10,13,14].

Denoting by $\Theta_{D}$ the moment of inertia of the "distinguishable" oscillators (i.e., without Bose-Einstein statistics), we also calculated the relative excess moment of inertia $\left(\Theta-\Theta_{D}\right) / \Theta_{D}$ numerically. The results are shown in Fig. 1 as a function of the reduced temperature for several values of $N$. For $N$ of order 100 or more the effect of the Bose statis- 


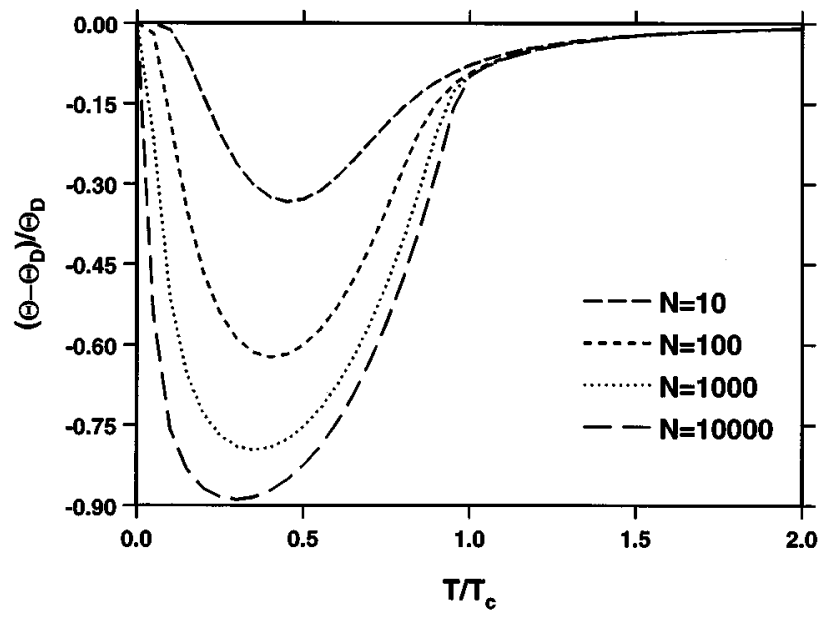

FIG. 1. Relative excess moment of inertia $\left(\Theta-\Theta_{D}\right) / \Theta_{D}$ with respect to the $z$ axis as a function of $T / T_{c}$ for $N=10,100,1000$, and 10000 .

tics on the moment of inertia is very pronounced, even reaching $75 \%$ for $N=1000$ and $90 \%$ for $N=10000$.

The Hellman-Feynman theorem is also applicable to energy estimates based on the variational principle of quantum mechanics $[16,17]$, and it can be used for models other than the isotropic gas of bosons in a parabolic well as considered here. For instance, by applying the Hellman-Feynman theorem using the internal energy from the semiclassical approximation [7], one finds the same moment of inertia as was derived by Stringari [4] from the rotational response properties of this semiclassical model. Note that the functions $Q(\eta)=\int_{0}^{\infty} s \eta /\left(e^{s}-1\right) d s$, used in Eqs. (9), (10), and (13) of Ref. [4], are related to the Riemann $\zeta$ functions $Q(2)=2 \zeta(3)$ and $Q(3)=6 \zeta(4)$ and are a special case of the well-known Bose-Einstein functions $g_{n}(z)$ $=\sum_{j=1}{ }^{\infty} z^{j} / j^{n}$, the properties of which are described in standard textbooks, e.g., in Ref. [18].

It should be emphasized that the Hellman-Feynman theorem is valid in the canonical as well as in the grand canonical ensemble. Because the internal energy in the grand canonical ensemble with an average number of $N$ bosons equals the internal energy in the canonical ensemble with a fixed number of $N$ bosons [19], also the moments of inertia of both ensembles are the same.

Having elucidated that the moment of inertia can be derived from the confinement energy in some models for the ideal or almost ideal gas, we did not discuss how quantitative results can be obtained in the presence of two-body interactions. Well aware of this limitation in the present paper, we would like to point out that the inclusion of harmonic twobody interactions presents no conceptual difficulty. Their influence on the partition function and on the thermodynamical properties can be and has been obtained [19] with a technique that we developed for treating the path integral for identical particles using the Feynman-Kac functional and invoking an appropriate linear combination of boson and fermion diffusion processes [20-22].

In summary, the partition function of an ideal Bose gas in a parabolic trap has been obtained in the present paper, and the ground-state occupancy $n_{0}$, the internal energy $U$, and the moment of inertia $\Theta$ have been derived. The rotational properties of the trapped gas are related to the internal energy through the Hellman-Feynman theorem. In the isotropic model we found the relation $\Theta=2 / 3\left(U / w^{2}\right)$, implying that the critical behavior of the internal energy will manifest itself macroscopically in the moment of inertia. The semiclassical approximation $[6,7,4]$ turns out to provide the correct asymptotic limits of this exactly soluble model. Moreover, the pathintegral method allows one to study this model as the zerothorder approximation in examining the role of the two-body interactions, using many-body diffusion and the FeynmanKac functional.

Part of this work was performed in the framework of NFWO Project Nos. 2.0093.91, 2.0110.91, G.0287.95, and WO.073.94N (Wetenschappelijke Onderzoeksgemeenschap, Scientific Research Community of the NFWO on "LowDimensional Systems"') and in the framework of the European Community Program Human Capital and Mobility through Contract Nos. CHRX-CT93-0337 and CHRX-CT930124. F.B. acknowledges the National Fund for Scientific Research for financial support.
[1] M. H. Anderson, J. R. Ensher, M. R. Matthews, C. E. Wieman, and E. A. Cornell, Science 269, 198 (1995).

[2] K. B. Davis, M. O. Mewes, M. R. Andrews, N. J. van Druten, D. S. Durfee, D. M. Kurn, and W. Ketterle, Phys. Rev. Lett. 75, 3969 (1995).

[3] C. C. Bradlet, C. A. Sacket, J. J. Tollett, and R. G. Hulet, Phys. Rev. Lett. 75, 1687 (1995).

[4] S. Stringari, Phys. Rev. Lett. 76, 1405 (1996).

[5] G. Baym, in Mathematical Methods in Solid State and Superfluid Theory, edited by R. C. Clark and E. H. Derrick (Oliver and Boyd, Edinburgh, 1969).

[6] S. A. De Groot, G. J. Hooyman, and A. Sedlam, Proc. R. Soc. London, Ser. A 203, 266 (1950).

[7] V. Bagnato, D. E. Pritchard, and D. Kleppner, Phys. Rev. A 35, 4354 (1987).
[8] M. Lewenstein, L. You, J. Cooper, and K. Burnett, Phys. Rev. A 50, 2287 (1994).

[9] S. Grossman and M. Holthaus, Z. Naturforsch. Teil A 50, 323, 921 (1995).

[10] S. Grossman and M. Holthaus, Phys. Lett. A 208, 188 (1995).

[11] W. Ketterle and N. J. van Druten, Phys. Rev. A 54, 656 (1996).

[12] K. Kirsten and D. J. Toms (unpublished).

[13] H. Haugerud, T. Haugset, and F. Ravndal (unpublished).

[14] H. D. Politzer (unpublished).

[15] R. P. Feynman, Statistical Mechanics: A Set of Lectures (Benjamin, Reading, MA, 1972), pp. 62 and 63.

[16] L. F. Lemmens, J. De Sitter, and J. T. Devreese, Phys. Rev. B 8, 2717 (1973). 
[17] L. F. Lemmens, F. Brosens, and J. T. Devreese, Phys. Rev. B 12, 4316 (1975).

[18] R. K. Pathria, Statistical Mechanics (Pergamon, Oxford, 1972), Appendix D.

[19] F. Brosens, J. T. Devreese, and L. F. Lemmens, Solid State Commun. 100, 123 (1996).
[20] L. F. Lemmens, F. Brosens, and J. T. Devreese, Phys. Lett. A 189, 437 (1994).

[21] F. Brosens, J. T. Devreese, and L. F. Lemmens, Solid State Commun. 96, 137 (1995).

[22] L. F. Lemmens, F. Brosens, and J. T. Devreese, Phys. Rev. E 53, 4467 (1996). 